\title{
Interstitial lung disease: a commentary
}

\author{
Esam H. Alhamad
}

Published online: 19 February 2013

(C) Springer Science+Business Media New York 2013

Keywords Autoimmune $\cdot$ Interstitial pneumonia $\cdot$ Idiopathic pulmonary fibrosis · Gastroesophageal reflux

Interstitial lung disease (ILD) is a heterogeneous group of disorders that diffusely affect the lung parenchyma and show variable etiologies, clinical presentations, radiographic patterns, and histological appearances. Together with asthma and chronic obstructive pulmonary disease, ILD is commonly encountered in clinical practice. Over the past decade, substantial progress has been made toward understanding many forms of ILD, particularly idiopathic interstitial pneumonia (IIP).

This commentary reviews: 1) the significance of positive serology for connective tissue disease (CTD) in patients with IIPs who do not fulfill the diagnostic criteria for any of the definite CTDs, and 2) the association between gastroesophageal reflux and idiopathic pulmonary fibrosis (IPF).

\section{Autoimmune and idiopathic interstitial pneumonias}

Many studies have shown that CTD associated with ILD carries a better prognosis than IIP, despite their similarities in pathological pattern and radiological severity [1-5]. While it can be straightforward to diagnose ILD in the presence of extrathoracic features of specific CTD, it can be more challenging to distinguish between autoimmune disease and IIP in the absence of overt systemic features of definite CTD. It has been estimated that up to $20 \%$ of cases

\footnotetext{
E. H. Alhamad $(\square)$

Interstitial lung Disease and Pulmonary Hypertension Center, Pulmonary Division, Department of Medicine (38), College of Medicine, King Saud University, P.O. Box 2925, Riyadh 11461, Saudi Arabia e-mail: esamalhamad@yahoo.com
}

of chronic ILD are either due to occult CTD or subsequently evolve into definite CTD [6, 7]. The 2011 guidelines on the diagnosis and management of IPF [8] recommend serological testing for CTD, including assays for antinuclear antibody (ANA), rheumatoid factor (RF), and anti-cyclic citrullinated peptide (anti-CCP). However, there are no reliable data on the role of serological screening when IPF is suspected.

Recent studies have attempted to address the clinical significance of ILD with autoimmune features that do not fulfill the diagnostic criteria for any specific CTD. In a retrospective study, Corte and colleagues [9] examined the significance of so-called "undifferentiated" CTD (UCTD) among IIP patients with surgically proven lung biopsies [45 patients with nonspecific interstitial pneumonia (NSIP) and 56 patients with IPF]. The authors found UCTD in $21 \%$ of their IIP patients, and observed that the diagnosis of UCTD was associated with a 3 -fold increase in the likelihood of NSIP. However, this was not sensitive or specific ( $31 \%$ and $88 \%$, respectively) for NSIP histology, and there did not appear to be any survival benefit compared to patients without UCTD.

Vij and colleagues [4] described autoimmune-featured ILD patients [usual interstitial pneumonia (UIP), NSIP, and unclassifiable] identified based on the presence of CTD symptoms and positive serological tests but insufficient extrathoracic features for a definite diagnosis of CTD. The authors compared these patients with IPF and CTD-ILD patients, and found that the 5-year survival rates were $95 \%$ for CTD-ILD, $48 \%$ for IPF, and $52 \%$ for autoimmunefeatured ILD. However, significantly improved survival was only noted among autoimmune-featured ILD patients with ANA titers greater than 1:1,280.

In another study, Alhamad and colleagues [3] prospectively described a group of patients that had UIP patterns and positive autoantibodies, but did not fulfill any of the definite forms of CTD; they called this lung-dominant (LD)- 
CTD, and compared it to IPF and CTD associated-UIP. In the LD-CTD group, no patient reported any systemic symptom or sign suggestive of CTD, either during the first lung manifestation of the disease or during an average follow-up of 3 years. The authors noted that the LD-CTD and CTDUIP patients were younger, more often female, and predominantly nonsmokers, compared with the IPF group. However, LD-CTD was not associated with improved survival when compared to the IPF group; the 3-year mortality rates for LD-CTD, CTD-UIP, and IPF were $30 \%, 16.7 \%$, and $33.3 \%$, respectively. Moreover, there was no difference in survival between LD-CTD patients with ANA titers greater than or equal 1,280 versus those with ANA titers less than 1,280 , suggesting that in the presence of the UIP pattern, ANA titer does not have prognostic significance.

Fischer and colleagues [10] reported 73 subjects with positive anti-CCP but no evidence of rheumatoid arthritis (RA) or other CTD. They identified four different HRCT patterns among these patients: $54 \%$ had isolated airway disease; $14 \%$ had isolated ILD; $26 \%$ had mixed airway disease and ILD; and $7 \%$ had combined pulmonary fibrosis and emphysema. The authors concluded that these HRCT patterns resembled those seen in patients with established RA and may represent a pre-RA phenotype. Interestingly, only three patients of their cohorts developed articular RA within 1.5 years of their initial pulmonary manifestations.

These studies illustrate that a detailed history, physical examination and serological testing may identify a subset of patients (of younger age and more often female) with CTD flavor (i.e., LD-CTD, autoimmune featured-ILD, or UCTD) among patients originally diagnosed as IIP. However, it appears that their outcomes do not differ from those of patients with IIPs. As such, large prospective studies will be needed to determine the true impact of positive serology in patients with IIP. Future studies will also be needed to identify reliable diagnostic criteria and biomarkers that can identify this group of patients early in their disease courses, and help determine the best treatment strategy given the underlying ILD (i.e., NSIP vs. UIP).

\section{Gastroesophageal reflux and idiopathic pulmonary fibrosis}

The association between gastroesophageal reflux (GER) and pulmonary fibrosis has long been reported, with microaspiration considered to be the likely underlying mechanism for pulmonary fibrosis [11]. However, the temporal relationship between the two is not yet clear, and it remains to be determined whether GER is a cause or effect of pulmonary fibrosis. Nonetheless, studies on the clinical relevance of GER in IPF have provided important information. Raghu and colleagues [12] reported a high prevalence of GER
$(87 \%)$ in IPF patients; $63 \%$ of them demonstrated proximal esophageal acid exposure, suggesting that aspiration may play a role in the pathogenesis of IPF. Tcherakian and colleagues [13] compared 32 patients with asymmetrical IPF (i.e., lung fibrosis involving one side more than the other) to 64 matched controls with symmetrical IPF. GER events were more frequent in asymmetrical than symmetrical IPF ( $62.5 \%$ vs. $31.3 \%$, respectively), and fibrosis was predominantly in the right side in $62.5 \%$ of patients with asymmetrical IPF. Interestingly, acute exacerbation was identified in $46.9 \%$ of patients with asymmetrical IPF compared to $17.2 \%$ with symmetrical IPF, suggesting that GER may play a role in exacerbation [13]. In another study, Lee and colleagues [14] measured pepsin as a biomarker for the presence of gastric aspiration in bronchoalveolar lavage (BAL) fluids from a cohort of IPF patients (30 with stable disease and 24 with acute exacerbation). The median level of BAL pepsin was higher in the acute exacerbation group compared to those with stable IPF, but these differences were not significant $\left(46.8 \mathrm{ng} \mathrm{ml}^{-1}\right.$ vs. $35.4 \mathrm{ng} \mathrm{ml}^{-1}, p=$ 0.15). However, the authors noted that an increase in BAL pepsin level equivalent to one standard deviation (24.8 $\mathrm{ng} \mathrm{ml}^{-1}$ ) was predictive of acute exacerbation, with an odds ratio of 1.46 (95\% CI $1.03-2.09 ; p=0.04)$. The authors concluded that the presence of pepsin in the BAL fluids of stable IPF patients supports the notion that microaspiration is common in IPF. They further concluded that the association of elevated BAL pepsin with acute exacerbation implies that occult aspiration may play a role in the pathogenesis of IPF, and could even be an important triggering factor for acute exacerbation. However, the findings of Lee and colleagues [14] should be interpreted with caution, because the use of a nonspecific antibody assay for pepsin may confound pepsin $\mathrm{C}$, which is type 2 cell-specific in the lung, with pepsin A, which is exclusive to the stomach. As such, a large prospective study is needed to identify an antibody assay that can reliably be used to predict the presence of nonacid aspiration.

One important question that arises from the above studies is: should acid suppression therapy be routinely prescribed to IPF patients? The current guidelines [8] recommended treating asymptomatic GER disease in IPF patients, but the expert panels acknowledged that the evidence reviewed during the preparation of the guidelines was of very low quality. After the guidelines were published, two intriguing retrospective studies explored the role of antireflux therapy in IPF patients.

In the first study, Lee and colleagues [15] noted that 96 of 203 patients receiving GER treatment were more likely to be women, have a history of cough, have GER symptoms, and have a lower HRCT fibrosis score. In the unadjusted analysis, they noted that a longer survival time was associated with female sex, higher pulmonary 
function test indices, presence of GER symptoms, and disease, use of GER medication, and a history of Nissen fundoplication. After adjustment, however, only three independent predictors were associated with longer survival time: a higher predicted percentage of forced vital capacity $(\mathrm{HR}=0.98$, $p=<0.01$ ), a higher predicted percentage of lung diffusion capacity for carbon monoxide $(\mathrm{HR}=0.98, p=0.03)$, and the use of GER medication (HR=0.47, $p=0.03$ ).

In the second study, Noth and colleagues [16] used multi detector computed tomography imaging to find a higher prevalence of hiatal hernia in IPF patients compared to those with asthma and COPD (39\%, $17 \%$, and $13 \%$, respectively). Among the IPF patients with hiatal hernias $(n=33)$ who were taking anti-reflux medication, they noted a significantly better lung diffusion capacity for carbon monoxide and composite physiological index score (as a measure of overall severity) compared to those who were not taking anti-reflux medication.

The results of these two studies further support the notion that microaspiration is an important pathway in the pathogenesis of IPF. However, a definitive conclusion cannot be drawn with regard to the beneficial effect of acid suppression therapy on disease progression or survival for the following reasons: 1) both studies were retrospective; 2) both studies examined small numbers of patients; 3) another study [12] found that $63 \%$ of IPF patients treated with proton pump inhibitors (20$40 \mathrm{mg}$ /day of omeprazole) at the time of the esophageal $\mathrm{pH}$ probe studies experienced persistently elevated esophageal acid exposure, implying that the current standard dose of acid suppression therapy does not adequately control or suppress the GER; and importantly, 4) antireflux medication does not eliminate the risk of aspirating nonacidic components of gastric juice, including bile acids, pepsin, trypsin and bacterial products.

Nonetheless, IPF is a devastating disease and no treatment has yet been shown to improve its outcome. Thus, a well-organized multicenter international prospective study is warranted to determine the impact of anti-reflux therapy (medication and surgery) on disease progression and survival.

Conflict of Interest Esam H. Alhamad declares no conflict of interest.

\section{References}

1. Park JH, Kim DS, Park IN, Jang SJ, Kitaichi M, Nicholson AG, et al. Prognosis of fibrotic interstitial pneumonia: idiopathic versus collagen vascular disease-related subtypes. Am J Respir Crit Care Med. 2007;175(7):705-11.

2. Song JW, Do KH, Kim MY, Jang SJ, Colby TV, Kim DS. Pathologic and radiologic differences between idiopathic and collagen vascular disease-related usual interstitial pneumonia. Chest. 2009; 136(1):23-30.

3. Alhamad EH, Al-Kassimi FA, Alboukai AA, Raddaoui E, Al-Hajjaj MS, Hajjar W, et al. Comparison of three groups of patients with usual interstitial pneumonia. Respir Med. 2012;106(11):1575-85.

4. Vij R, Noth I, Strek ME. Autoimmune-featured interstitial lung disease: a distinct entity. Chest. 2011;140(5):1292-9.

5. Flaherty KR, Colby TV, Travis WD, Toews GB, Mumford J, Murray S, et al. Fibroblastic foci in usual interstitial pneumonia: idiopathic versus collagen vascular disease. Am J Respir Crit Care Med. 2003;167(10):1410-5.

6. Strange $\mathrm{C}$, Highland KB. Interstitial lung disease in the patient who has connective tissue disease. Clin Chest Med. 2004;25(3):549-59.

7. Homma Y, Ohtsuka Y, Tanimura K, Kusaka H, Munakata M, Kawakami Y, et al. Can interstitial pneumonia as the sole presentation of collagen vascular diseases be differentiated from idiopathic interstitial pneumonia? Respiration. 1995;62(5):248-51.

8. Raghu G, Collard HR, Egan JJ, Martinez FJ, Behr J, Brown KK, et al. An official ATS/ERS/JRS/ALAT statement: idiopathic pulmonary fibrosis: evidence-based guidelines for diagnosis and management. Am J Respir Crit Care Med. 2011;183(6):788-824.

9. Corte TJ, Copley SJ, Desai SR, Zappala CJ, Hansell DM, Nicholson AG, et al. Significance of connective tissue disease features in idiopathic interstitial pneumonia. Eur Respir J. 2012;39(3):661-8.

10. Fischer A, Solomon JJ, du Bois RM, Deane KD, Olson AL, Fernandez-Perez ER, et al. Lung disease with anti-CCP antibodies but not rheumatoid arthritis or connective tissue disease. Respir Med. 2012;106(7):1040-7.

11. Mays EE, Dubois JJ, Hamilton GB. Pulmonary fibrosis associated with tracheobronchial aspiration. A study of the frequency of hiatal hernia and gastroesophageal reflux in interstitial pulmonary fibrosis of obscure etiology. Chest. 1976;69(4):512-5.

12. Raghu G, Freudenberger TD, Yang S, Curtis JR, Spada C, Hayes J, et al. High prevalence of abnormal acid gastro-oesophageal reflux in idiopathic pulmonary fibrosis. Eur Respir J. 2006;27(1):136-42.

13. Tcherakian C, Cottin V, Brillet PY, Freynet O, Naggara N, Carton $Z$, et al. Progression of idiopathic pulmonary fibrosis: lessons from asymmetrical disease. Thorax. 2011;66(3):226-31.

14. Lee JS, Song JW, Wolters PJ, Elicker BM, King Jr TE, Kim DS, et al. Bronchoalveolar lavage pepsin in acute exacerbation of idiopathic pulmonary fibrosis. Eur Respir J. 2012;39(2):352-8.

15. Lee JS, Ryu JH, Elicker BM, Lydell CP, Jones KD, Wolters PJ, et al. Gastroesophageal reflux therapy is associated with longer survival in patients with idiopathic pulmonary fibrosis. Am J Respir Crit Care Med. 2011;184(12):1390-4.

16. Noth I, Zangan SM, Soares RV, Forsythe A, Demchuk C, Takahashi $\mathrm{SM}$, et al. Prevalence of hiatal hernia by blinded multidetector CT in patients with idiopathic pulmonary fibrosis. Eur Respir J. 2012;39(2):344-51. 